\title{
EMBALAGEM INDIVIDUAL DE MANGAS CV. TOMMY ATKINS EM FILME PLÁSTICO: EFEITO SOBRE A VIDA DE PRATELEIRA ${ }^{1}$
}

\author{
FÁBIO YAMASHITA², ANAMARIA CALDO TONZAR ${ }^{3}$; JOICELENA GEORGETTI FERNANDES; \\ SUELY MORIYA ${ }^{3}$, MARTA DE TOLEDO BENASSI ${ }^{2}$
}

\begin{abstract}
RESUMO - Estudou-se o efeito da embalagem de policloreto de vinila (PVC) sobre a vida de prateleira de mangas cv. Tommy Atkins armazenadas sob refrigeração. Mangas no estádio de maturidade fisiológica, com casca verde ou levemente avermelhada, foram embaladas individualmente, com filme de $10 \mathrm{~mm}$ de espessura, e armazenadas por 28 dias a $12^{\circ} \mathrm{C}(80-90 \% \mathrm{UR})$. Frutos sem embalagem serviram de controle. Durante o período de armazenagem, foram feitas avaliações sensoriais utilizando o método de escala hedônica não estruturada para aceitação da aparência e do sabor, utilizando-se de 30 provadores não treinados por sessão. Determinou-se também a perda de massa, a acidez titulável e os teores de sólidos solúveis e vitamina $\mathrm{C}$ ao longo da armazenagem. As mangas embaladas apresentaram uma vida de prateleira de 21 dias contra 6 dias das não embaladas, e uma taxa de perda de massa 3,5 vezes menor que as não embaladas. Em relação à taxa de degradação de vitamina $\mathrm{C}$, não houve diferença entre os tratamentos. A combinação da embalagem com a armazenagem a $12^{\circ} \mathrm{C}$ aumentou a vida de prateleira do produto pela redução da atividade metabólica e do desenvolvimento de podridão.
\end{abstract}

Termos para indexação: Mangifera indica L., pós-colheita, análise sensorial, vitamina C

\section{INDIVIDUAL PACKAGING OF MANGOS CV. TOMMY ATKINS IN PLASTIC FILM: EFFECT ON SHELF LIFE}

\begin{abstract}
Effects of packaging in polyvinyl chloride (PVC) film on postharvest shelf-life of mango cv. Tommy Atkins stored under refrigeration were studied. Mangos at mature green color stage were individually sealed in $10 \mathrm{~mm}$ thick film and stored for 28 days at $12^{\circ} \mathrm{C}(80-90 \% \mathrm{RH})$. Non-sealed fruits served as control. During the storage period, sensory evaluation was carried out using an unstructured hedonic scale for overall acceptance of appearance and flavor, with 30 untrained assessors per session. Mangos were also analyzed for weight loss, titratable acidity, total soluble solids and vitamin C during storage time. The packed mangos had a shelf life of 21 days against 6 days of the control ones, and weight loss rates 3.5 times smaller than the control ones. There was no difference in vitamin $\mathrm{C}$ degradation rate between treatments. The combination of packaging with storage at $12^{\circ} \mathrm{C}$ was efficient to extend shelf life by reducing metabolic activity and decay in mangos.
\end{abstract}

Index terms: Mangifera indica L., postharvest, sensory evaluation, vitamin C.

\section{INTRODUÇÃO}

O Brasil é um grande produtor de manga, mas o volume exportado ainda é pequeno, devido principalmente à curta vida de prateleira do produto que dificulta o transporte marítimo. A embalagem de frutos em filmes plásticos diminui as taxas de respiração, transpiração e crescimento microbiano e outras reações metabólicas que ocorrem no produto, através da criação e manutenção de uma micro-atmosfera ótima (Ben-Yehoshua, 1985; Zagory \& Kader, 1988; Yamashita et al., 1997a). Entretanto, uma combinação de embalagem e temperatura inadequada poderá causar uma entrada de oxigênio e/ou saída de gás carbônico insuficientes através da embalagem, criando uma atmosfera não apropriada ao fruto, podendo provocar desde alterações de cor e textura até o desenvolvimento de sabor e odor não característicos (Miller et al., 1986; Yamashita et al., 1997b). A literatura cita o emprego de diferentes filmes plásticos (polietileno, polipropileno, diversos copolímeros de alta permeabilidade) para embalar individualmente mangas de diversas cultivares, como 'Tommy Atkins' (Miller et al., 1983 e 1986), 'Palmer' (Melo Neto et al., 1999) e 'Keitt' (Yamashita et al., 1997a). O filme de policloreto de vinila (PVC) tem baixo custo e alta permeabilidade a gases e foi utilizado por Jeronimo \& Kanesiro (2000) para recobrir bandejas contendo manga cv. 'Palmer'. O objetivo deste trabalho foi verificar a influência da embalagem individual de PVC sobre algumas características sensoriais e físico-químicas de mangas cv. Tommy Atkins mantidas sob refrigeração, tendo em vista o aumento da vida de prateleira das mesmas.

\section{MATERIAL E MÉTODOS}

Os experimentos foram realizados no Laboratório de Tecnologia de Frutas do Departamento de Engenharia e Tecnologia de Alimentos da UNESP, Câmpus de São José do

1 Trabalho ${ }^{\circ}$ 030/2001. Recebido: 06/02/2001. Aceito para publicação: 27/06/2001.

2 Professores, Depto. Tecnologia de Alimentos e Medicamentos, Universidade Estadual de Londrina, C.P. 6001, CEP: 86051-970, Londrina, PR. Email: fabioy@uel.br.

3 Alunas Iniciação Científica, DETA/UNESP, C.P. 136, CEP 15054-000, S. J. Rio Preto, SP. 
Rio Preto.

Foram utilizados $75 \mathrm{~kg}$ de mangas cv Tommy Atkins, no estádio de maturidade fisiológica, casca verde ou levemente avermelhada, provenientes da região de S. J. Rio Preto-SP, com massa média de $572 \pm 56 \mathrm{~g}$. Os frutos fora dos padrões de massa e estádio de maturação foram descartados, assim como os que apresentavam doenças e injúrias mecânicas.

Para a embalagem dos frutos, foi utilizado um filme de PVC transparente de $10 \mathrm{~mm}$. As amostras foram embaladas individualmente, colocando-se apenas uma camada do filme sobre o fruto. As mangas foram imersas em água clorada 100 ppm por 15 minutos, embaladas, codificadas, e armazenadas a $12^{\circ} \mathrm{C} \pm 1{ }^{\circ} \mathrm{C} / 80-90 \%$ UR, sem pré-resfriamento, em câmara frigorífica dotada de sistema de ventilação, por 28 dias. Mangas sem embalagem serviram de controle ou testemunha. Utilizouse em torno de 60 frutos para cada tratamento

Foram determinados, de acordo com as normas analíticas do Instituto Adolfo Lutz (1985), a acidez total titulável (\% ácido cítrico) e teor de sólidos solúveis totais ( $\left.{ }^{\circ} \mathrm{Brix}\right)$ da polpa tanto dos frutos armazenados como daqueles utilizados na análise sensorial de sabor. As análises foram realizadas a cada 6-7 dias, ao longo da armazenagem, utilizando três frutos por análise.

$\mathrm{A}$ análise de vitamina $\mathrm{C}$ foi realizada ao longo do período de armazenagem de acordo com o método titulométrico oficial da AOAC (1985) modificado por Benassi \& Antunes (1988). Através de regressão linear, com os valores do logaritmo neperiano do teor de vitamina $\mathrm{C}$ pelo tempo de armazenagem, foram calculados a taxa de degradação de vitamina $\mathrm{C}$ e o tempo de meia-vida, conforme Labuza (1982).

Para o estudo da perda de massa, quatro mangas de cada tratamento, escolhidas aleatoriamente, foram pesadas em balança semi-analítica a cada sete dias ao longo do período de armazenagem. Foi feita uma regressão linear dos resultados em função do tempo, para se determinar à taxa de perda de massa de cada tratamento.

A cada 6-7 dias, um lote de 10 frutos de cada tratamento era retirado da refrigeração e colocado para amadurecer, à temperatura ambiente $\left(25-30^{\circ} \mathrm{C} / 75-95 \% \mathrm{UR}\right)$, sem embalagem, para evitar o desenvolvimento de sabor não característico, observado por Miller et al. (1983 e 1986) em mangas Tommy Atkins que amadureceram com filmes plásticos. O amadurecimento foi verificado pelos analistas, após tempos variáveis de armazenagem ambiente, através de avaliação visual, por pressão com os dedos e desenvolvimento de aroma característico nos frutos. Após o amadurecimento, 30 provadores não treinados, consumidores do produto, avaliavam a aparência dos frutos e o sabor de suas polpas, por meio de uma escala hedônica não estruturada de $9 \mathrm{~cm}(0=$ desgostei muitíssimo, $9=$ gostei muitíssimo) (Stone \& Siedel, 1993). A avaliação de aceitação em função do sabor foi realizada em cabines com luz branca, à temperatura ambiente. Três cubos de polpa de manga (com $3 \mathrm{~cm}^{3}$ ) foram servidos em prato plástico descartável. A avaliação de aceitação com relação à aparência foi feita fora das cabines. Os frutos foram retirados da câmara frigorífica e ficaram expostos em cima de uma bancada com boa iluminação, onde os provadores pudessem observá-los, sem a embalagem. Os provadores preencheram uma ficha individual de avaliação para cada amostra, tanto para aparência como para sabor. $\mathrm{O}$ fím da vida de prateleira foi determinado quando o fruto apresentou sinais aparentes de deterioração ou obteve nota sensorial inferior a 6,0, conforme recomendado por Labuza (1982).

Para a comparação da aceitação das amostras submetidas aos diferentes trata-mentos, foram feitas análises de variância e aplicou-se o Teste de Tukey (Bhattacharyya \& Johnson, 1977). Para a determinação dos coeficientes e parâmetros estatísticos das regressões lineares, foi utilizado o programa StatisticaÒ 5.0 (Statsoft, 1995).

\section{RESULTADOS E DISCUSSÃO}

Ao longo da armazenagem a $12^{\circ} \mathrm{C}$, foi feito o acompanhamento da acidez total titulável (ATT) e do teor de sólidos solúveis totais (SST) dos frutos (Figura 1). Em termos de ATT, as mangas não apresentaram um comportamento diferenciado entre os tratamentos. Tanto as mangas embaladas como as sem embalagem apresentaram um aumento no teor de SST em função do tempo; entretanto, os frutos-controle apresentaram um aumento maior $\left(12\right.$ a $17^{\circ}$ Brix $)$ que os embalados (12 a $14^{\circ}$ Brix), constatando-se que a embalagem reduziu a atividade metabólica do fruto, retardando o seu amadurecimento. A relação SST/ATT, no final do período de armazenamento, foi de 23,3 para os frutos-controle e 16,5 para mangas embaladas. Salles \& Tavares (1999), trabalhando com armazenamento $\left(10^{\circ} \mathrm{C}\right.$ por 30 dias) de mangas 'Tommy Atkins', observaram aumento menos pronunciado de sólidos solúveis e maior redução na acidez; a relação SST/AAT apresentou uma grande variação em função dos diferentes estádios de maturação (10 a 80).

Após 28 dias de armazenagem, as perdas de massa foram de, aproximadamente, $20 \mathrm{~g} / 100 \mathrm{~g}$ e $6 \mathrm{~g} / 100 \mathrm{~g}$ para as mangascontrole e embaladas, respectivamente, e seguiram uma cinética de ordem zero, correspondendo a uma taxa de desidratação constante, o que é admissível em processos com grande resistência na interface (Figura 2). O uso de embalagem reduziu de forma significativa a taxa de perda de massa das mangas, pois a taxa calculada para mangas sem embalagem foi aproximadamente 3,5 vezes maior em relação às embaladas (Tabela 1).

A manga pode ser considerada uma boa fonte de vitamina $\mathrm{C}$, devido à concentração encontrada inicialmente no fruto $(36 \pm 2 \mathrm{mg} / 100 \mathrm{~g})$. No Brasil, a ingestão diária recomendada (IDR) de vitamina C para adultos é de 60mg (Brasil, 1998). Após 28 dias de armazenagem, os frutos apresentaram teores de $22 \pm 7 \mathrm{mg} / 100 \mathrm{~g}$ e $19 \pm 5 \mathrm{mg} / 100 \mathrm{~g}$, no controle e embalados, respectivamente. As curvas do teor de vitamina $\mathrm{C}$ em função do tempo de armazenagem configuraram um perfil sugestivo de uma reação de primeira ordem, tanto para mangas embaladas como para as não embaladas (Figura 3).

Os frutos embalados apresentaram taxa de degradação de vitamina $\mathrm{C}$ em torno de 1,25 maior que os não embalados (Tabela 2), entretanto não foi observada diferença significativa entre os valores. Jeronimo e Kanesiro (2000), trabalhando com mangas 'Palmer' embaladas com diversos tipos de filmes plásticos, também não observaram diferença entre mangas com e sem embalagem em termos de degradação de vitamina $C$. Yamashita et al. (1999), trabalhando com mangas cv. Keitt com diferentes embalagens (D-955 e PEBD), observaram taxas de degradação de vitamina $\mathrm{C}$ semelhantes, em torno de $2 \mathrm{mg}$ / 
100g.dia. Os mesmos autores verificaram que as embalagens reduziram a taxa de degradação, comparadas com o controle, quando os frutos foram armazenados a temperaturas mais altas $\left(17\right.$ e $\left.22^{\circ} \mathrm{C}\right)$, mas não observaram este efeito à $12^{\circ} \mathrm{C}$.

Com relação à avaliação sensorial, as mangas com e sem embalagem após o $21^{\circ}$ e $13^{\circ}$ dias de armazenagem, respectivamente, tornaram-se impróprias para o consumo devido ao desenvolvimento de podridão por antracnose e não puderam ser avaliadas em termos de sabor. Em todos os tratamentos, houve problemas com desenvolvimento de podridão por antracnose e alguns trabalhos da literatura têm proposto o emprego de tratamento fitossanitário, como a utilização de fungicida (Salles \& Tavares, 1999) ou imersão em água quente (Jeronimo \& Kanesiro, 2000).

Após 13 dias de armazenagem, as mangas embaladas com PVC obtiveram notas de aparência superiores àquelas sem embalagem, que já estavam impróprias para comercialização, pois obtiveram uma nota média de aparência baixa $(2,7)$, causada pelo enrugamento e perda de brilho da casca e início do desenvolvimento de podridão, apesar de estarem com sabor aceitável. Já os frutos embalados obtiveram, até o $21^{\circ}$ dia de armazenagem, notas médias de sabor e aparência superiores a 6
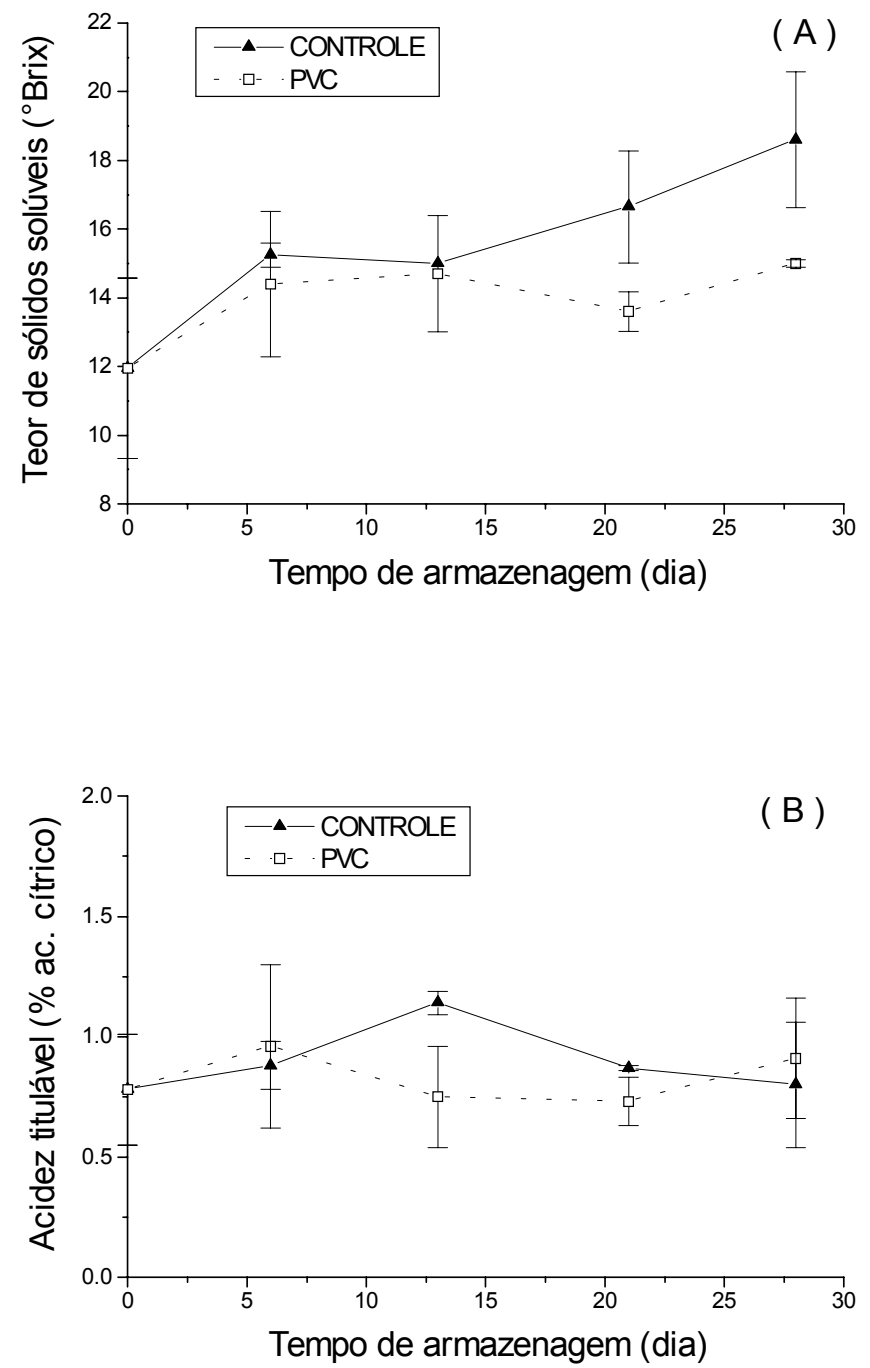

FIGURA 1. Variação do teor de sólidos solúveis totais (A) e acidez total titulável (B) das mangas cv. Tommy Atkins em função do tempo de armazenagem a $12^{\circ} \mathrm{C}$.
(Tabela 3).

Para a aceitação em termos de sabor, não foi observada diferença entre as notas obtidas pelas mangas embaladas com PVC e sem embalagem ao longo do período de armazenagem. Já as notas de aparência apresentaram um declínio em função do tempo de armazenagem, ou seja, o fim da vida de prateleira foi regido pela aparência (Tabela 3).

As análises físico-químicas das mangas maduras utilizadas na análise sensorial de sabor indicaram que, tanto as mangas embaladas como as controle, conseguiram amadurecer normalmente até 21 e 13 dias de armazenagem a $12^{\circ} \mathrm{C}$, respectivamente (Tabela 4). Pode-se observar que, para todos os tempos, a maturação provocou um aumento no teor de sólidos solúveis e redução na acidez titulável e no teor de vitamina $\mathrm{C}$, comparando-se esses valores aos apresentados nas Figuras 1 e 3 (mangas não maduras).

Aparentemente, a embalagem não causou injúria fisiológica nas mangas que impedisse o seu amadurecimento normal e nem foi detectado pelos provadores o desenvolvimento de sabor não característico.

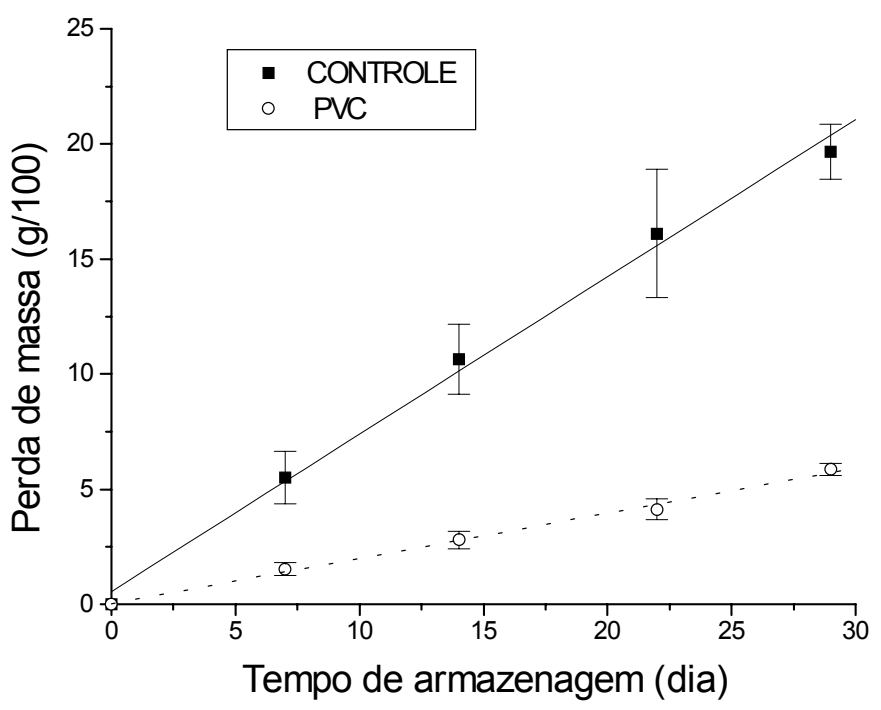

FIGURA 2. Perda de massa de mangas cv. Tommy Atkins em função do tempo de armazenagem a $12^{\circ} \mathrm{C} \pm 1{ }^{\circ} \mathrm{C} /$ $80-90 \%$ UR.

TABELA 1. Valores de taxa de perda de massa $\left(\mathrm{N}_{\mathrm{m}}\right)$, coeficiente de determinação $\left(\mathrm{r}^{2}\right)$ e nível de significância $(\mathrm{p})$ das correlações para perda de massa de mangas cv. Tommy Atkins armazenadas a $12^{\circ} \mathrm{C} \pm 1{ }^{\circ} \mathrm{C} / 80$ $90 \%$ UR.

\begin{tabular}{cccc}
\hline EMBALAGEM & $\mathrm{N}_{\mathrm{m}}$ (g/kg.dia) & $\mathrm{r}^{2}$ & $\mathrm{p}(\%)$ \\
\hline CONTROLE & $6,84^{\mathrm{a}}$ & 0,99 & 0,02 \\
PVC & $1,96^{\mathrm{b}}$ & 1,00 & 0,21 \\
\hline $\mathrm{a}, \mathrm{b}$ médias com a mesma letra não diferem significativamente, ao nível de $5 \%$.
\end{tabular}


TABELA 2. Taxa de degradação de vitamina $\mathrm{C}\left(\mathrm{N}_{\mathrm{vc}}\right)$, tempo de meia-vida $\left(\mathrm{t}_{1 / 2}\right)$, coeficiente de determinação ( $\left.\mathrm{r}^{2}\right)$ e nível de significância das correlações (p) de mangas cv. Tommy Atkins armazenadas a $12^{\circ} \mathrm{C} \pm 1{ }^{\circ} \mathrm{C} / 80-90 \%$ UR.

\begin{tabular}{|c|c|c|c|c|c|c|}
\hline E $m b$ a 1 a g e $m$ & 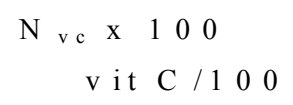 & $\mathrm{g} \cdot \mathrm{dia})^{(\mathrm{m} g}$ & $\mathrm{t}_{1 / 2}$ & $(\mathrm{~d}$ i a s ) & $r^{2}$ & $\mathrm{p} \quad(\%)$ \\
\hline $\mathrm{C} O \mathrm{O}$ T R O L E & 1,76 & a & & 100 & 0,93 & 0,72 \\
\hline $\mathrm{P} \vee \mathrm{C}$ & 2,21 & a & & 79 & 0,92 & 1,03 \\
\hline
\end{tabular}

a,b médias com a mesma letra não diferem significativamente, ao nível de $5 \%$.

TABELA 3. Notas da avaliação sensorial de sabor e aparência das mangas cv. Tommy Atkins sem embalagem e embaladas com filme de $\mathrm{PVC}$, armazenadas a $12^{\circ} \mathrm{C} \pm 1^{\circ} \mathrm{C} / 80-90 \% \mathrm{UR}$ e amadurecidas à temperatura ambiente $\left(25-30^{\circ} \mathrm{C} / 75-95 \% \mathrm{UR}\right)$.

\begin{tabular}{|c|c|c|c|c|}
\hline T E M P O & TRATA M EN TO & \multicolumn{3}{|c|}{ NO TA M É D IA* } \\
\hline (dia) & & $S A B O R$ & \multicolumn{2}{|c|}{ A PARÊ N CIA } \\
\hline 0 & CONTROLE & 7,1 a & 8,8 & a \\
\hline \multirow[t]{2}{*}{6} & CONTROLE & 6,5 a & 5,4 & b c \\
\hline & $\mathrm{P} \vee \mathrm{C}$ & 6,0 a & 5,7 & b c \\
\hline \multirow[t]{2}{*}{13} & CONTROLE & 6,6 a & 2,7 & $d$ \\
\hline & $P \vee C$ & 6,8 a & 6,2 & $b$ \\
\hline \multirow[t]{2}{*}{21} & CONTROLE & i.c. & 3,1 & $d$ \\
\hline & $\mathrm{P} \vee \mathrm{C}$ & 6,4 a & 6,6 & $b$ \\
\hline \multirow[t]{2}{*}{28} & CONTROLE & i.c. & i.c. & \\
\hline & $P \vee C$ & i.c. & 4,5 & c \\
\hline
\end{tabular}

*Nota $(0=$ desgostei muitíssimo, 9 = gostei muitíssimo)

i.c. - impróprio para consumo

${ }^{a, b}$ médias da mesma coluna, com a mesma letra, não diferem significativamente entre si, ao nível de 5\%.

TABELA 4. Teor de sólidos solúveis totais (SST), vitamina C e acidez total titulável (ATT) das mangas maduras cv. Tommy Atkins utilizadas na avaliação sensorial de sabor

\begin{tabular}{ccccc}
\hline $\begin{array}{c}\text { TEMPO } \\
(\text { dia })\end{array}$ & TRATAMENTO & SST & A T T & Vitam in C \\
\hline 0 & CON TROLE & $17,20 \pm 1,29$ & $0,24 \pm 0,07$ & $(\mathrm{mg} / 100 \mathrm{~g})$ \\
\hline 6 & C O N TROLE & $18,33 \pm 2,15$ & $0,23 \pm 0,08$ & $27,10 \pm 2,06$ \\
\hline 13 & PVC & $17,83 \pm 1,89$ & $0,27 \pm 0,06$ & $21,08 \pm 6,57$ \\
\hline 21 & C O N TRO LE & $18,93 \pm 1,56$ & $0,51 \pm 0,12$ & $17,29 \pm 0,68$ \\
\hline & PVC & $17,30 \pm 2,05$ & $0,27 \pm 0,10$ & $14,45 \pm 2,36$ \\
\hline
\end{tabular}




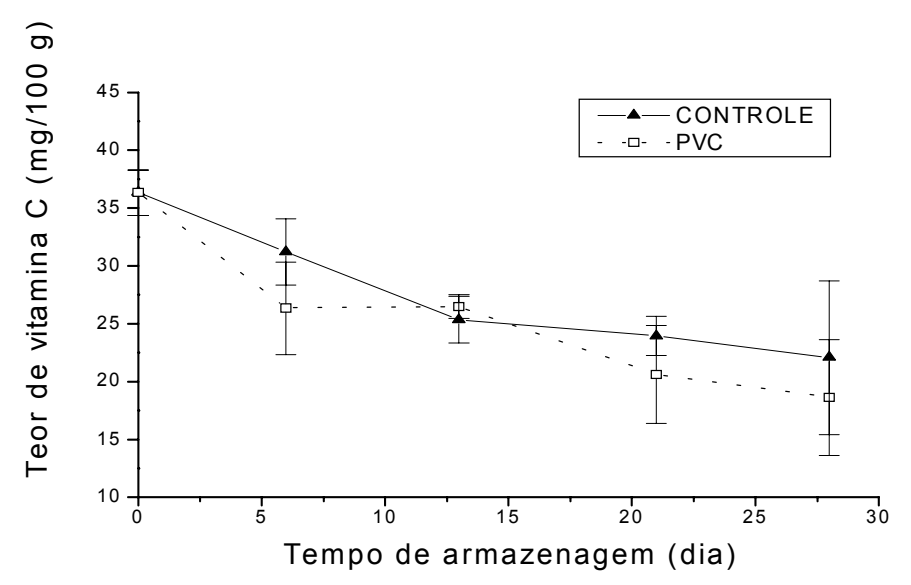

FIGURA 3. Variação do teor de vitamina $\mathrm{C}$ em mangas cv. Tommy Atkins em função do tempo de armazenagem a $12^{\circ} \mathrm{C} \pm 1^{\circ} \mathrm{C} / 80-90 \%$ UR.

\section{CONCLUSÕES}

A combinação da embalagem individual, em filme de PVC com a armazenagem a $12^{\circ} \mathrm{C}$, mostrou-se eficaz para conservação pós-colheita de mangas cv. Tommy Atkins, prolongando a vida de prateleira (21 dias contra 6 dias dos frutos sem embalagem), sem ter causado injúria fisiológica que afetasse os frutos em termos de sabor e aparência, além de ter reduzido em 3,5 vezes a taxa de perda de massa. Este aumento da vida útil foi devido à redução da atividade metabólica e do desenvolvimento de podridão nas mangas.

\section{REFERÊNCIAS BIBLIOGRÁFICAS}

AOAC Official methods of analysis. Washington, D. C: Association of Official Analytical Chemists, 1984. p. 844-845. BENASSI, M.T.; ANTUNES, A.J.A. Comparison of metaphosphoric and oxalic acids as extractant solutions for the determination of vitamin $C$ in selected vegetables. Arquivos de Biologia e Tecnologia, Curitiba, v.31, n.4, p.507-513, 1988.

BHATTACHARYYA, G.K.; JOHNSON, R.A. Statistical concepts and methods. New York: John Wiley \& Sons, 1977. $639 \mathrm{p}$.

BRASIL. Portaria SVS/MS n. ${ }^{\text {o }}$ 33, de 13 de janeiro de 1998. Tabelas de Ingestão Diária Recomendada IDR. Diário Oficial da União de 16 de janeiro de 1998. Seção 1, pt 1.

INSTITUTO ADOLFO LUTZ. Normas analíticas do Instituto Adolfo Lutz: métodos químicos e físicos para análise dos alimentos. 3.ed. São Paulo: Editora do Instituto Adolfo Lutz, 1985, v.1.371p.
JERONIMO, E.M.; KANESIRO, M.A.B. Efeito da associação de armazenamento sob refrigeração e atmosfera modificada na qualidade de mangas "Palmer". Revista Brasileira de Fruticultura, Jaboticabal, v.22, n.2, p.237-243, 2000.

BEN-YEHOSHUA, S. Individual seal-packaging of fruit and vegetables in plastic film - a new postharvest technique. HortScience, Alexandria, v.20, n.1, p.32-37, 1985.

LABUZA, T.P. Shelf-life dating of foods. Westport:: Food \& Nutrition Press Inc., 1982.

MELO NETO, M.L.; CHRISTOFFOLETI, P.J.; SIGRIST, J.M.M.; ALVES, R.M.V. Utilização de embalagens plásticas e refrigeração na conservação da manga (Mangifera indica L.) cv. Palmer. Revista Brasileira de Fruticultura, Jaboticabal, v.21, n.2, p.160-165, 1999.

MILLER, W. R.; HALE, P. W.; SPALDING, D. H.; DAVIS, P. Quality and decay of mango fruit wrapped in heat-shrinkable film. HortScience, Alexandria, v.18, n.6, p.957-958, 1983.

MILlER, W. R.; SPALDING, D. H.; HALE, P. W. Film wrapping mangos at advancing stages of post-harvest ripening. Tropical Science, London, v.26, n.1, p.9-17, 1986.

SALLES, J.R.J.; TAVARES, J.C. Vida útil pós-colheita de manga (Mangifera indica L. cv. Tommy Atkins): influência da temperatura e do estádio de maturação. Revista Brasileira de Fruticultura, Jaboticabal, v.21, n.2, p.171-176, 1999.

STATSOFT STATISTICA for Windows - Computer program manual. Tulsa: Statsoft, 1995.

STONE, H.; SIDEL, J.L. Sensory evaluation practices. 2.ed. Orlando: Academic Press, 1993.

YAMASHITA, F.; BENASSI, M.T.; KIECKBUSCH, T.G. Shelf life extension of individually film-wrapped mangoes. Tropical Science, London, v.37, p.249-255, 1997a.

YAMASHITA, F.; TELIS-ROMERO, J.; KIECKBUSCH, T. G. Estimativa da composição gasosa em embalagem de atmosfera modificada contendo mangas (Mangifera indica L.) cv. Keitt. Ciência e Tecnologia de Alimentos, Campinas, v.17, n.2, p.172176, $1997 b$.

YAMASHITA, F.; BENASSI, M. T.; KIECKBUSCH, T. G. Effect of modified atmosphere packaging on kinetics of vitamin $\mathrm{C}$ degradation in mangos. Brazilian Journal of Food Technology, Campinas, v.2, n.1-2, p.127-130, 1999.

ZAGORY, D.; KADER, A.A. Modified atmosphere packaging of fresh produce. Food Technology, Chicago, v.42, n.9, p.7074, 76-77, 1988. 